\title{
Evaluación de fertilizantes de liberación controlada con fertilización convencional, sobre el rendimiento de maíz duro (zea mays) en la zona de Febres-Cordero, Provincia de los Ríos
}

\author{
Evaluation of controlled-release fertilizers with conventional fertilization on the yield of \\ hard maize (zea mays) in Febres-Cordero, Province of Los Ríos
} Avaliação de fertilizantes de liberação controlada com adubação convencional sobre o
rendimento de milho duro (zea mays) na área de Febres-Cordero, província de Ríos

Eduardo Colina ${ }^{1}$

ed-colina@hotmail.com

Carlos Castro ${ }^{1}$

Hugo Sánchez ${ }^{1}$

Germán Troya ${ }^{2}$

${ }^{1}$ Universidad Técnica de Babahoyo, Ecuador

${ }^{2}$ Técnico Plan Maíz-MAGAP, Ministerio de Agricultura y Ganadería, Ecuador

Artículo recibido mayo 2017, arbitrado junio 2017 y publicado en septiembre 2017

\section{RESUMEN}

El presente estudio está dedicado a destacar la importancia que tienen el uso de fertilizantes de liberación controlada los cuales juegan un papel importante en la mejora del rendimiento de los cultivos, reduciendo las pérdidas de nutrientes y facilitando la aplicación de fertilizantes para un mejor rendimiento en el maíz duro. Método: se utilizó como material vegetativo los híbridos de maíz DK-7088 y H-601. Las aplicaciones se realizaron en presencia de la humedad de suelo. En los tratamientos solo con fertilizantes de liberación controlada se realizó una solo aplicación 5 días después de la siembra. Los resultados muestran que el uso de fertilizantes de liberación controlada solos o en mezclas con fertilización química convencional, influencian el rendimiento del cultivo de maíz en la zona de estudio. Los rendimientos presentados fueron muy aceptables, alcanzando su tope más alto con $7854.2 \mathrm{~kg} / \mathrm{ha}$ en promedio siendo el hibrido de maíz DK-7088 (9041.7 kg/ha) superior a la producción del híbrido H-601 $(6666.7 \mathrm{~kg} / \mathrm{ha})$. Sin embargo los resultados alcanzados con la utilización de fertilizantes de liberación controlada solos, no superan a la mezcla de estos con fertilización convencional.

\begin{abstract}
The present study is dedicated to emphasize the importance of the use of controlled release fertilizers, which play an important role in improving crop yields, reducing nutrient losses and facilitating the application of fertilizers for better yield hard corn Method: DK7088 and H-601 maize hybrids were used as vegetative material. Applications were made in the presence of soil moisture. In treatments with only controlled release fertilizers, a single application was per-formed 5 days after sowing. The results show that the use of controlled release fertilizers alone or in mixtures with conventional chemical fertilization, influence the yield of corn in the study area. The yields presented were very acceptable, reaching their highest level with $7854.2 \mathrm{~kg} /$ ha on average being the corn hybrid DK-7088 (9041.7 kg / ha) higher than the production of the hybrid $\mathrm{H}-601$ (6666.7 kg / ha). However, the results achieved with the use of controlled release fertilizers alone, do not exceed the mixture of these with conventional fertilization.
\end{abstract}

Key words: Fertilizer controlled; yield; conventional; maize; biomass

Palabras clave: Fertilizante controlados; rendimiento; convencional; maíz; biomasa 


\section{RESUMO}

O presente estudo dedica-se a destacar a importância do uso de fertilizantes de liberação controlada, que desempenham um papel importante na melhoria do rendimento das culturas, reduzindo as perdas de nutrientes e facilitando a aplicação de fertilizantes para obter um melhor rendimento no milho duro Método: Híbridos de milho DK-7088 e H-601 foram utilizados como material vegetativo. As aplicações foram feitas na presença de umidade do solo. Nos tratamentos apenas com fertilizantes de liberação controlada, apenas uma aplicação foi realizada 5 dias após a semeadura. Os resultados mostram que o uso de fertilizantes de liberação controlada isoladamente ou em misturas com fertilização química convencional influencia o rendimento da safra de milho na área de estudo. Os rendimentos apresentados foram muito aceitáveis, atingindo seu pico mais alto com 7854,2 kg / ha em média, o híbrido de milho DK-7088 (9041,7 kg / ha) excedendo a produção do híbrido H-601 (6666,7 kg / ha). No entanto, os resultados alcançados com o uso de fertilizantes de liberação controlada isoladamente não excedem a mistura destes com a fertilização convencional.

Palavras-chave: Fertilizante controlado; Desempenho; convencional; milho; biomassa

\section{INTRODUCCIÓN}

El maíz (Zea mays. L.) es un cultivo de mucha importancia económica para el país, se siembra en la costa bajo diferentes condiciones ambientales de temperatura, humedad, régimen de lluvias, luminosidad y suelos. El maíz duro en Ecuador constituye la principal materia prima para la elaboración de alimentos balanceados. El rendimiento promedio del maíz en el litoral ecuatoriano es bajo, debido principalmente al empleo de un deficiente manejo tecnológico, pues existen híbridos cuyo rendimiento puede superar en el orden del 30 al $60 \%$ en comparación a los rendimientos obtenidos por las variedades cuando se lo siembra con tecnología (Sierra, 2007).

La aplicación de fertilizantes hace que a corto plazo se obtengan muy buenos resultados en producción, pero a largo plazo, los efectos negativos en el medio ambiente y en la salud humana han sido catastróficos. Hasta el punto que una cantidad de estos productos han sido prohibidos y muchos otros se encuentran en procesos de ser prohibidos (Trenkel, 2007) También, el uso inadecuado y continuo de ellos ha hecho que el equilibrio en el recurso suelo, logrando que se pierda la eficiencia de los mismos, lo que repercute en baja producción. Por este proceso aparece el uso de los fertilizantes de liberación gradual, controlada o lenta.

La fertilización es un factor decisivo en los cultivos y determinan los siguientes objetivos económicos: a) Reducción de costos; b) Aumento del beneficio por unidad de superficie y por unidad de fertilizante aplicado. Los efectos en el cultivo y su relación con los objetivos económicos determinan los puntos a seguir en lo referente a dosis, tipos de fertilizantes y su forma de aplicación de acuerdo a las condiciones reales de la explotación agrí́cola (INIAP, 2008).

La aplicación de fertilizantes convencionales ha hecho que en el corto plazo se obtengan muy buenos resultados en producción. Sin embargo su efecto a largo plazo por su mal uso ha ocasionado efectos negativos en el medio ambiente y en la salud del recurso suelo, siendo estos en algunos casos catastróficos. También, el uso inadecuado y continuo de ellos ha hecho que el equilibrio en la rizósfera del suelo se pierda, bajando consigo la eficiencia de los mismos, lo que repercute en baja producción. Por este proceso aparece el uso de los 
fertilizantes de liberación gradual, controlada o lenta (Trenkel, 2007).

Los fertilizantes de liberación controlada juegan un papel importante en la mejora del rendimiento del cultivo, reduciendo las pérdidas de nutrientes $\mathrm{y}$ facilitando la aplicación de fertilizantes. La razón es sencilla: la tecnología de liberación controlada sincroniza el suministro de nutrientes con las necesidades de la planta. Una planta joven, por ejemplo, sólo tolera un bajo nivel de nutrientes, mientras que las plantas maduras requieren un mayor aporte de los mismos (Tucunango, 1993).

Los fertilizantes de liberación controlada en si son productos que precisan de solo una única aplicación a lo largo de todo el ciclo para proveer la cantidad necesaria de nutrientes para una producción óptima. Además de necesitarse una única aplicación para lograr el máximo retorno económico del insumo, y deben tener un mínimo efecto negativo sobre el ambiente: el suelo, el agua y la atmósfera (Melgar, 2012).

Los fertilizantes de liberación controlada juegan un papel importante en la mejora del rendimiento de los cultivos, reduciendo las pérdidas de nutrientes $y$ facilitando la aplicación de fertilizantes. Esto se debe a que la tecnología de liberación controlada sincroniza el suministro de nutrientes con las necesidades (fisiológica y morfología) de la planta. Una planta joven, por ejemplo, sólo tolera un bajo nivel de nutrientes, mientras que las plantas maduras requieren un mayor aporte de los mismos. (Tucunango, 1993).

La fertilización balanceada también ocupa un rol importante. La misma tiene que apuntar a la reposición de los nutrientes extraídos por las cosechas, contribuyendo a su vez a elevar el contenido de materia orgánica del suelo. El suelo agrícola configura el soporte más sólido de la economía de los países y conservarlo se torna imprescindible para garantizar el bienestar de todos los habitantes, por esto la importancia de proteger los suelos productivos, verdadera fábrica de alimentos (Thompson, $2002 \mathrm{y}$ Casas, 2011).

La búsqueda de nuevas alternativas de fertilización que complementarias a un manejo agronómico adecuado, constituye una de las prioridades actuales en el manejo de suelos a nivel mundial.

Novatec $₫$ es un fertilizante de liberación controlada que se encuentra cubierto con resina de poliuretano que hace que los nutrientes se liberen continua $y$ controladamente. Baja los costos de aplicación ya que no requiere de aplicaciones adicionales. Por su alta calidad y mayor rendimiento, las plantas consiguen cantidades óptimas de nutrientes todos los días, estas se encuentran muy saludables. Normalmente requiere solo una aplicación (Agrociencias, 2013).

Así mismo Multisuelo® es un fertilizante de liberación gradual que contiene arcillas especiales y materia orgánica de alta capacidad de intercambio catiónico que retienen los nutrientes (especialmente los más móviles en el suelo como el Amonio y el Potasio), lo cual minimiza su lixiviación y maximiza su eficacia o absorción a la planta. La materia orgánica contenida en Multisuelo® mejora la disponibilidad del fósforo y aumenta su traslocación al cultivo. Todos estos beneficios técnicos que brinda Multisuelo® se traducen en una mayor eficiencia de fertilización (mejor resultado técnico económico) en comparación con los fertilizantes tradicionales (Enlasa, 2013).

\section{MATERIALES Y MÉTODOS}

El presente trabajo de investigación se realizó en los terrenos de la hacienda "Macondo", ubicada en el recinto "El tigrillo", perteneciente a la parroquia Febres Cordero, Cantón Babahoyo. Se localiza a $68 \mathrm{~km}$ de la ciudad de Babahoyo, con coordinadas 
geográficas de $157^{\prime} 22^{\prime \prime}$ de latitud sur 79 o $17^{\prime} 35^{\prime \prime}$ de la capital oeste. La zona presenta un clima tropical húmedo, con una temperatura anual de 25.7 o C, una precipitación anual de $2100 \mathrm{~mm}$, humedad relativa de $86 \%$ y 804.7 horas heliofanía de promedio anual, Suelo profundo alfisol, altitud $45 \mathrm{msnm}$.

Se utilizó como material vegetativo los híbridos de maíz DK-7088 y H-601. Las aplicaciones se realizaron en presencia de la humedad de suelo. En los tratamientos solo con fertilizantes de liberación controlada se realizó una solo aplicación 5 días después de la siembra. En los tratamientos con mezclas y programas solos se realizaron tres aplicaciones a los 5-20-40 días después de la siembra. La primera aplicación se realizó el 5 de mayo del 2014 y las restantes el 20 de mayo y la ultima el 10 de junio del 2014.

Se probaron en campo los siguientes tratamientos:

Novatec

Multisuelo

Fertilización Química (FQ)

Fertilización Agricultor (FA)

F.Q. $50 \%+25 \%$ Novatec

F.A. $50 \%+25 \%$ Novatec

F.Q. $50 \%+25 \%$ Multisuelo

F.A. $50 \%+25 \%$ Multisuelo

Para el desarrollo y evaluación estadística del ensayo se utilizó el diseño "Bloques Completo al Azar" (BCA) en parcelas divididas con 8 subtratamientos y 3 repeticiones. La comparación y evaluación de las medias, se realizó con la prueba de Tukey al $99 \%$ de probabilidad.

El cultivo se sembró bajo labranza cero, en parcelas rectangulares $3 \times 4 \mathrm{~m}$ aplicando riego a los 50 días después de la siembra. Las evaluaciones de las variables agronómicas se llevaron a cabo según criterios de desarrollo.
Se evaluó: altura de planta, altura de inserción, numero de mazorcas por plantas, días a floración, días a maduración, longitud de mazorcas, diámetro de mazorcas y rendimiento por hectárea.

El control de gusano cogollero (Spodoptera frujiperda) se realizó con Cipermetrina ( $0.3 \mathrm{~L} / \mathrm{ha}$ ) a los 20 días después de la siembra y a 42 días después de la siembra con Clorpirifos (1 L/ha). Se utilizó Diazinon $1 \mathrm{~L} /$ ha para el control del chupador Dalbolus. Para el control de mancha de asfalto (Monographella y Phyllacora) se aplicó 0.3 L/ha de Amistar top a los 50 días después de la siembra.

Las malezas se controlaron con la Pendimentalin $2 \mathrm{~L} / \mathrm{ha}$, Amina 0,5 L/ha $\mathrm{y}$ Atrazina $1 \mathrm{~kg} / \mathrm{ha}$, en preemergencia. Posteriormente se aplicó $1 \mathrm{~L} /$ ha de Paraquat. Los análisis estadísticos fueron llevados a cabo por medio del programa Stafgraff.

\section{RESULTADOS Y DISCUSIÓN}

\section{Altura de planta}

En los promedios de altura de planta Se observó alta significancia estadística para híbridos, Fertilizantes e interacciones. El coeficiente de variación fue de $3.19 \%$.

El híbrido DK-7088 presentó la mayor altura $(219.45 \mathrm{~cm})$, siendo estadísticamente superior a INIAP H-601 l híbrido que logró 202.87 cm. La aplicación de F.Q. $50 \%+112$ $\mathrm{kg} / \mathrm{ha}$ de Multisuelo registró la mayor altura $245.16 \mathrm{~cm}$. El menor registro se dio la fertilización del agricultor con $196.16 \mathrm{~cm}$.

En las interacciones entre híbridos y fertilizantes, se observó la mayor altura con la aplicación de F.Q. $50 \%+112 \mathrm{~kg} / \mathrm{ha}$ de Multisuelo $(262.67 \mathrm{~cm}$ ) en el hibrido DK-7088 y la menor altura con Fertilización Agricultor en el híbrido DK-7088 (192.33 cm). (Cuadro 1). 
Cuadro 1. Altura de planta de maíz con la aplicación fertilizantes edáficos en mezcla con fertilizantes de liberación controlada. Babahoyo.

\begin{tabular}{|c|c|c|c|c|c|c|c|c|c|}
\hline \multicolumn{10}{|c|}{$\begin{array}{c}\text { Subtratamientos (Fertilizantes) } \\
\text { Altura de plantas }(\mathrm{cm})\end{array}$} \\
\hline $\begin{array}{l}\text { Tratamientos } \\
\text { (Híbridos) }\end{array}$ & $\begin{array}{c}\text { Novatec } \\
400 \mathrm{~kg} / \mathrm{ha}\end{array}$ & $\begin{array}{c}\text { Multisuelo } \\
100 \%\end{array}$ & $\begin{array}{l}\text { Fertilización } \\
\text { Química }\end{array}$ & $\begin{array}{l}\text { Fertilización } \\
\text { Agricultor }\end{array}$ & $\begin{array}{l}\text { F.A. } 50 \%+25 \% \\
\quad \text { Novatec }\end{array}$ & $\begin{array}{l}\text { F.A. } 50 \%+25 \% \\
\quad \text { Novatec }\end{array}$ & F.A. $50 \%+25 \%$ & $\begin{array}{l}\text { F.A. } 50 \%+25 \% \\
\text { Multisuelo }\end{array}$ & $\begin{array}{l}\text { Promedio } \\
\text { (Híbridos (**) }\end{array}$ \\
\hline DK-7088 & 212.00 & 213.33 & 215.67 & 192.33 & 226.00 & 213.00 & 262.67 & 219.45 & 219.45 a \\
\hline INIAP H-601 & 201.00 & 198.67 & 193.33 & 193.00 & 201.00 & 197.67 & 227.67 & 204.67 & $202.87 \mathrm{~b}$ \\
\hline $\begin{array}{l}\text { Promedio } \\
\text { Fertilizantes } \\
(* *)\end{array}$ & $206.50 \mathrm{bc}$ & $206.00 \mathrm{bc}$ & $204.50 \mathrm{bc}$ & $195.16 \mathrm{c}$ & $213.50 \mathrm{bc}$ & $205.33 \mathrm{bc}$ & $245.16 \mathrm{a}$ & 212.16 bc & \\
\hline
\end{tabular}

**: Altamente significante al $5 \%$.

Valores promedios con la misma letra no difieren estadísticamente según prueba de Tukey al 5\%.

\section{Número de mazorcas}

El número de mazorcas por planta no encontró significancia estadística para tratamientos, subtratamientos e interacciones, siendo el coeficiente de variación de $3.76 \%$.

Dk-7088 (1.29 mazorca/planta) reportó el mayor número de mazorcas, obteniéndose el menor promedio con INIAP H-601 con 1.33 mazorcas/planta. Se evidenció el mayor número de mazorcas por planta (2.16) con F.Q. $50 \%+112 \mathrm{~kg} /$ ha de Multisuelo. Menores promedios se presentaron con Multisuelo 100 \% (1.00 mazorcas/planta). La interacción F.Q. $50 \%+112 \mathrm{~kg} / \mathrm{ha}$ de Multisuelo con DK-7088 registró el mayor número de mazorcas con 2.33. El menor número de mazorcas (0.90) se tuvo en el híbrido H-601cuando se aplicó F.Q. $50 \%+112 \mathrm{~kg} /$ ha de Novatec (ver cuadro 2).

Cuadro 2. Número de mazorcas por planta con la aplicación fertilizantes edáficos en mezcla con fertilizantes de liberación controlada. Babahoyo Días a floración

\begin{tabular}{|c|c|c|c|c|c|c|c|c|c|}
\hline \multicolumn{10}{|c|}{$\begin{array}{c}\text { Subtratamientos (Fertilizantes) } \\
\text { Altura de plantas }(\mathrm{cm})\end{array}$} \\
\hline $\begin{array}{l}\text { Tratamientos } \\
\text { (Híbridos) }\end{array}$ & $\begin{array}{l}\text { Novatec } \\
400 \mathrm{~kg} / \mathrm{ha}\end{array}$ & $\begin{array}{l}\text { Multisuelo } \\
460 \mathrm{~kg} / \mathrm{ha}\end{array}$ & $\begin{array}{l}\text { Fertilización } \\
\text { Química }\end{array}$ & $\begin{array}{c}\text { Fertilización } \\
\text { Agricultor }\end{array}$ & $\begin{array}{l}\text { F.A. } 50 \%+25 \% \\
\text { Novatec }\end{array}$ & $\begin{array}{l}\text { F.A. } 50 \%+25 \% \\
\text { Novatec }\end{array}$ & $\begin{array}{c}\text { F.A. } 50 \%+25 \% \\
\text { Multisuelo }\end{array}$ & $\begin{array}{l}\text { F.A. } 50 \%+25 \% \\
\text { Multisuelo }\end{array}$ & $\begin{array}{l}\text { Promedio } \\
\text { Híbridos (Ns) }\end{array}$ \\
\hline DK-7088 & 1.00 & 1.00 & 1.33 & 1.00 & 1.33 & 1.33 & 2.33 & 1.00 & 1.29 \\
\hline INIAP H-601 & 1.33 & 1.00 & 1.33 & 1.33 & 0.90 & 1.33 & $2 \ldots$ & 1.33 & 1.33 \\
\hline $\begin{array}{l}\text { Promedio } \\
\text { Fertilizantes } \\
(\mathrm{Ns})\end{array}$ & 1.16 & 1.00 & 1.33 & 1.16 & 1.16 & 1.33 & 2.16 & 1.16 & \\
\hline
\end{tabular}

Coeficiente de variación (\%): 3.76

Alfa, Revista de Investigación en Ciencias Agronómicas y Veterinarias Vol. 1, JNo. 3, Septiembre-Diciembre 2017 
Los promedios de días a floración obtenidos tuvieron alta significancia estadística para fertilizantes e interacción híbridos $\mathrm{x}$ fertilizante. El coeficiente de variación presente fue de $1.89 \%$.

DK-7088 (52.83 días) tuvo el mayor promedio, el menor promedio se dio con INIAP H-601 (52.72 días). En los subtratamientos fertilizantes se registró que la utilización de F.Q. $50 \%+112 \mathrm{~kg} / \mathrm{ha}$ de Novatec (57.20 días) fue estadísticamente superior a los demás tratamientos. El menor número de días se obtuvo con la aplicación de Novatec $400 \mathrm{~kg} / \mathrm{ha}$ (49.50 días). En las interacciones entre híbridos y fertilizantes, se observó que el mayor número de días se encontró en el hibrido H-601 con F.A. $50 \%$ + $112 \mathrm{~kg} / \mathrm{ha}$ Novatec dio 57.67 días. La floración más temprana se manifestó en $\mathrm{H}$ 601 con Novatec $400 \mathrm{~kg} / \mathrm{ha}$ con 48.67 días (Cuadro 3).

Cuadro 3. Días a la floración con la aplicación fertilizantes edáficos en mezcla con fertilizantes de liberación controlada. Babahoyo.

\begin{tabular}{|c|c|c|c|c|c|c|c|c|c|}
\hline \multicolumn{10}{|c|}{$\begin{array}{c}\text { Subtratamientos (Fertilizantes) } \\
\text { Altura de plantas }(\mathrm{cm})\end{array}$} \\
\hline $\begin{array}{l}\text { Tratamientos } \\
\text { (Híbridos) }\end{array}$ & $\begin{array}{c}\text { Novatec } \\
400 \mathrm{~kg} / \mathrm{ha}\end{array}$ & $\begin{array}{l}\text { Multisuelo } \\
460 \mathrm{~kg} / \mathrm{ha}\end{array}$ & $\begin{array}{l}\text { Fertilización } \\
\text { Química }\end{array}$ & $\begin{array}{c}\text { Fertilización } \\
\text { Agricultor }\end{array}$ & $\begin{array}{l}\text { F.A. } 50 \%+25 \% \\
\quad \text { Novatec }\end{array}$ & $\begin{array}{l}\text { F.A. } 50 \%+25 \% \\
\text { Novatec }\end{array}$ & $\begin{array}{c}\text { F.A. } 50 \%+25 \% \\
\text { Multisuelo }\end{array}$ & $\begin{array}{l}\text { F.A. } 50 \%+25 \% \\
\text { Multisuelo }\end{array}$ & $\begin{array}{c}\text { Promedio } \\
\text { Híbridos (Ns) }\end{array}$ \\
\hline DK-7088 & 50.33 & 50.37 & 52.00 & 53.00 & 54.33 & 56.67 & 50.27 & 50.10 & 52.83 \\
\hline INIAP H-601 & 48.67 & 50.97 & 50.33 & 53.47 & 55.33 & 57.67 & 50.17 & 50.06 & 52.72 \\
\hline $\begin{array}{l}\text { Promedio } \\
\text { Fertilizantes } \\
(* *)\end{array}$ & $49.5 \mathrm{~d}$ & $50.7 \mathrm{~cd}$ & $51.2 \mathrm{~cd}$ & $53.3 \mathrm{bc}$ & $54.8 \mathrm{ab}$ & $57.2 \mathrm{a}$ & $50.2 \mathrm{~cd}$ & $50.1 \mathrm{~cd}$ & \\
\hline
\end{tabular}

Coeficiente de variación (\%): 1.89

Ns, no significante en subtratamientos.

**: Altamente significante al $5 \%$.

\section{Días a maduración}

La maduración fisiológica de la planta no presentó significancia estadística para híbridos, pero se logró para fertilizantes e interacción híbridos $\mathrm{x}$ fertilizante. El coeficiente de variación presente fue de 1.91 $\%$.

INIAP H-601 (109.84 días) tuvo el mayor promedio, el menor promedio se dio con DK7088 (52.72 días). Los subtratamientos fertilizantes dieron mayor número de días con F.Q. $50 \%+112 \mathrm{~kg} / \mathrm{ha}$ de Novatec $(120.3$ días) el cual fue estadísticamente superior a los demás tratamientos. Menor número de días se dio con la aplicación de Novatec 400 $\mathrm{kg} / \mathrm{ha}$ (104.5 días). Las interacciones híbridos y fertilizantes, mostraron mayor número de días en el hibrido H-601 con F.A. $50 \%+112$ $\mathrm{kg} /$ ha Novatec con 121.33 días, dando la floración más temprana en H-601 con Novatec $400 \mathrm{~kg} / \mathrm{ha}$ con 102.33 días (Cuadro 4). 
Cuadro 4. Días a la maduración fisiológica con la aplicación fertilizantes edáficos en mezcla con fertilizantes de liberación controlada. Babahoyo.

\begin{tabular}{|c|c|c|c|c|c|c|c|c|c|}
\hline \multicolumn{10}{|c|}{$\begin{array}{c}\text { Subtratamientos (Fertilizantes) } \\
\text { Altura de plantas }(\mathrm{cm})\end{array}$} \\
\hline $\begin{array}{l}\text { Tratamientos } \\
\text { (Híbridos) }\end{array}$ & $\begin{array}{c}\text { Novatec } \\
400 \mathrm{~kg} / \mathrm{ha}\end{array}$ & $\begin{array}{l}\text { Multisuelo } \\
460 \mathrm{~kg} / \mathrm{ha}\end{array}$ & $\begin{array}{c}\text { Fertilización } \\
\text { Química }\end{array}$ & $\begin{array}{c}\text { Fertilización } \\
\text { Agricultor }\end{array}$ & $\begin{array}{l}\text { F.A. } 50 \%+25 \% \\
\quad \text { Novatec }\end{array}$ & $\begin{array}{l}\text { F.A. } 50 \%+25 \% \\
\quad \text { Novatec }\end{array}$ & $\begin{array}{l}\text { F.A. } 50 \%+25 \% \\
\text { Multisuelo }\end{array}$ & $\begin{array}{l}\text { F.A. } 50 \%+25 \% \\
\text { Multisuelo }\end{array}$ & $\begin{array}{l}\text { Promedio } \\
\text { Híbridos (Ns) }\end{array}$ \\
\hline DK-7088 & 105.67 & 106.33 & 107.00 & 113.33 & 114.33 & 119.33 & 106.00 & 104.2 & 109.52 \\
\hline INIAP H-601 & 102.33 & 106.33 & 107.67 & 110.33 & 116.67 & 121.33 & 104.4 & 108.0 & 109.84 \\
\hline $\begin{array}{l}\text { Promedio } \\
\text { Fertilizantes }(* *) \\
\text { Coeficiente de var }\end{array}$ & $\begin{array}{r}104.0 \mathrm{~d} \\
\text { riación (\%) }\end{array}$ & $106.3 \mathrm{~cd}$ & $107.3 \mathrm{~cd}$ & $111.8 \mathrm{bc}$ & $115.5 \mathrm{ab}$ & $120.3 \mathrm{a}$ & $105.2 \mathrm{~cd}$ & $106.1 \mathrm{~cd}$ & \\
\hline
\end{tabular}

Ns, no significante en subtratamientos.

**: Altamente significante al $5 \%$.

Valores promedios con la misma letra no difieren estadísticamente según prueba de Tukey al 5\%.

\section{Longitud de mazorcas}

El número de mazorcas por planta no encontró significancia estadística para híbridos, si para fertilizantes e interacciones, siendo el coeficiente de variación de $8.56 \%$.

Dk-7088 $(17.16 \mathrm{~cm})$ presentó la mayor longitud de mazorcas, menor promedio dio INIAP H-601 con $16.95 \mathrm{~cm}$. Cuando se utilizó F.Q. $50 \%+112 \mathrm{~kg} / \mathrm{ha}$ de Multisuelo se observó mayor longitud de mazorcas (22.8 $\mathrm{cm})$, siendo superior estadísticamente a los demás tratamientos. La interacción F.Q. $50 \%$ + 112 kg/ha de Multisuelo con DK-7088 registró el mayor longitud con 23.33, menor tamaño se dio con la fertilización del agricultor (14.33) con el uso de H-601 (Cuadro 5).

Cuadro 5. Longitud de mazorcas de maíz con la aplicación fertilizantes edáficos en mezcla con fertilizantes de liberación controlada. Babahoyo.

\begin{tabular}{|c|c|c|c|c|c|c|c|c|c|}
\hline \multicolumn{10}{|c|}{$\begin{array}{c}\text { Subtratamientos (Fertilizantes) } \\
\text { Longitud }(\mathrm{cm})\end{array}$} \\
\hline $\begin{array}{l}\text { Tratamientos } \\
\text { (Híbridos) }\end{array}$ & $\begin{array}{c}\text { Novatec } \\
400 \mathrm{~kg} / \mathrm{ha}\end{array}$ & $\begin{array}{l}\text { Multisuelo } \\
460 \mathrm{~kg} / \mathrm{ha}\end{array}$ & $\begin{array}{c}\text { Fertilización } \\
\text { Química }\end{array}$ & $\begin{array}{c}\text { Fertilización } \\
\text { Agricultor }\end{array}$ & $\begin{array}{l}\text { F.A. } 50 \%+25 \% \\
\text { Novatec }\end{array}$ & $\begin{array}{c}\text { F.A. } 50 \%+25 \% \\
\text { Novatec }\end{array}$ & $\begin{array}{l}\text { F.A. } 50 \%+25 \% \\
\text { Multisuelo }\end{array}$ & $\begin{array}{l}\text { F.A. } 50 \%+25 \% \\
\text { Multisuelo }\end{array}$ & $\begin{array}{c}\text { Promedio } \\
\text { Híbridos (Ns) }\end{array}$ \\
\hline DK-7088 & 16.67 & 16.00 & 15.67 & 16.33 & 17.33 & 15.67 & 23.33 & 16.33 & 17.16 \\
\hline INIAP H-601 & 16.00 & 16.00 & 16.67 & 14.33 & 16.67 & 16.33 & 22.33 & 17.33 & 16.95 \\
\hline $\begin{array}{l}\text { Promedio } \\
\text { Fertilizantes }\left(^{* *}\right) \\
\text { Coeficiente de va }\end{array}$ & $\begin{array}{l}16.3 \mathrm{bc} \\
\text { riación (\%): } 8\end{array}$ & $16.0 \mathrm{bc}$ & $16.2 \mathrm{bc}$ & $15.3 \mathrm{c}$ & $17.0 \mathrm{bc}$ & $16.0 \mathrm{bc}$ & $22.8 \mathrm{a}$ & $16.8 \mathrm{bc}$ & \\
\hline
\end{tabular}

Ns, no significante en subtratamientos.

**: Altamente significante al $5 \%$.

Valores promedios con la misma letra no difieren estadísticamente según prueba de Tukey al 5\%. 
Evaluación de fertilizantes de liberación controlada con fertilización convencional, sobre el rendimiento de maíz duro (zea mays) en la zona de Febres-Cordero, Provincia de los Ríos

\section{Rendimiento por hectárea}

El rendimiento de grano por hectárea encontró alta significancia estadística para híbridos, fertilizantes e interacciones, siendo el coeficiente de variación de $4.41 \%$.

Dk-7088 (6953.1 kg/ha) presentó el rendimiento más alto, menor promedio dio INIAP H-601 con $5822.9 \mathrm{~kg} / \mathrm{ha}$. Cuando se utilizó F.Q. $50 \%+112 \mathrm{~kg} / \mathrm{ha}$ de Multisuelo se observó incremento del rendimiento con7854.2 $\mathrm{kg} / \mathrm{ha}$, siendo superior estadísticamente a los demás tratamientos. La interacción F.Q. $50 \%+112 \mathrm{~kg} / \mathrm{ha}$ de Multisuelo con DK-7088 registró mayor producción con $9041.7 \mathrm{~kg} / \mathrm{ha}$, menor rendimiento se dio con la fertilización F.A. 50 $\%+25 \%$ Novatec $(5000,00 \mathrm{~kg} / \mathrm{ha})$ con el uso de H-601(Cuadro 6).

Cuadro 6. Rendimiento por hectárea de maíz con la aplicación fertilizantes edáficos en mezcla con fertilizantes de liberación controlada. Babahoyo.

\begin{tabular}{|c|c|c|c|c|c|c|c|c|c|}
\hline \multicolumn{10}{|c|}{$\begin{array}{c}\text { Subtratamientos (Fertilizantes) } \\
\text { Altura de plantas }(\mathrm{cm})\end{array}$} \\
\hline $\begin{array}{l}\text { Tratamientos } \\
\text { (Híbridos) }\end{array}$ & $\begin{array}{c}\text { Novatec } \\
400 \mathrm{~kg} / \mathrm{ha}\end{array}$ & $\begin{array}{l}\text { Multisuelo } \\
460 \mathrm{~kg} / \mathrm{ha}\end{array}$ & $\begin{array}{c}\text { Fertilización } \\
\text { Química }\end{array}$ & $\begin{array}{c}\text { Fertilización } \\
\text { Agricultor }\end{array}$ & $\begin{array}{l}\text { F.A. } 50 \%+25 \% \\
\quad \text { Novatec }\end{array}$ & $\begin{array}{c}\text { F.A. } 50 \%+25 \% \\
\text { Novatec }\end{array}$ & $\begin{array}{l}\text { F.A. } 50 \%+25 \% \\
\text { Multisuelo }\end{array}$ & $\begin{array}{l}\text { F.A. } 50 \%+25 \% \\
\text { Multisuelo }\end{array}$ & $\begin{array}{c}\text { Promedio } \\
\text { Híbridos (**) }\end{array}$ \\
\hline DK-7088 & 6750,0 & 6916,7 & 6375,0 & 5791,7 & 7000,0 & 6416,7 & 9041,7 & 7333,3 & $6953.1 \mathrm{a}$ \\
\hline INIAP H-601 & 5916,7 & 6041,7 & 5916,7 & 5791,7 & 5333,3 & 5000,0 & 6666,7 & 5916,7 & $5822.9 \mathrm{~b}$ \\
\hline $\begin{array}{l}\text { Promedio } \\
\text { Fertilizantes }(* *) \\
\text { Coeficiente de vari }\end{array}$ & $\begin{array}{l}6333.3 \text { bc } \\
\text { riación (\%): } 4\end{array}$ & $6749.2 \mathrm{~b}$ & $6145.8 \mathrm{bc}$ & 5791.7 c & $6166.7 \mathrm{bc}$ & $5708.3 \mathrm{c}$ & $7854.2 \mathrm{a}$ & $6625.0 \mathrm{~b}$ & \\
\hline
\end{tabular}

Ns, no significante en subtratamientos.

**: Altamente significante al $5 \%$.

Valores promedios con la misma letra no difieren estadísticamente según prueba de Tukey al 5\%.

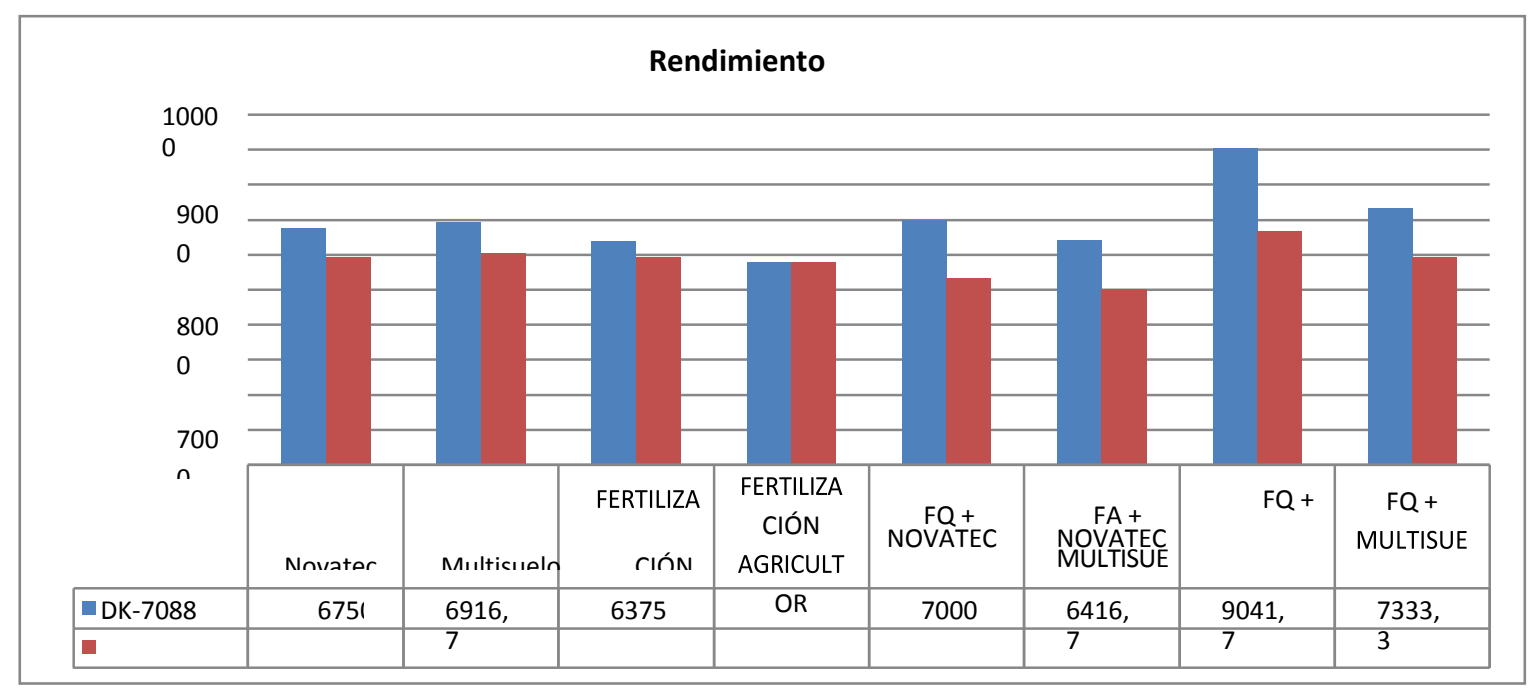

Figura 1. Rendimiento por hectárea con la aplicación fertilizantes edáficos en mezcla con fertilizantes de liberación controlada. Babahoyo. 


\section{Correlaciones}

El estudio determino la existencia de alta correlación entre las variables evaluadas las mismas fueron: rendimiento por altura de planta, rendimiento por longitud de mazorca y altura de planta por longitud de mazorca. Las factores de correlación fueron: 0.81, 0.64 y 0.67, respectivamente.

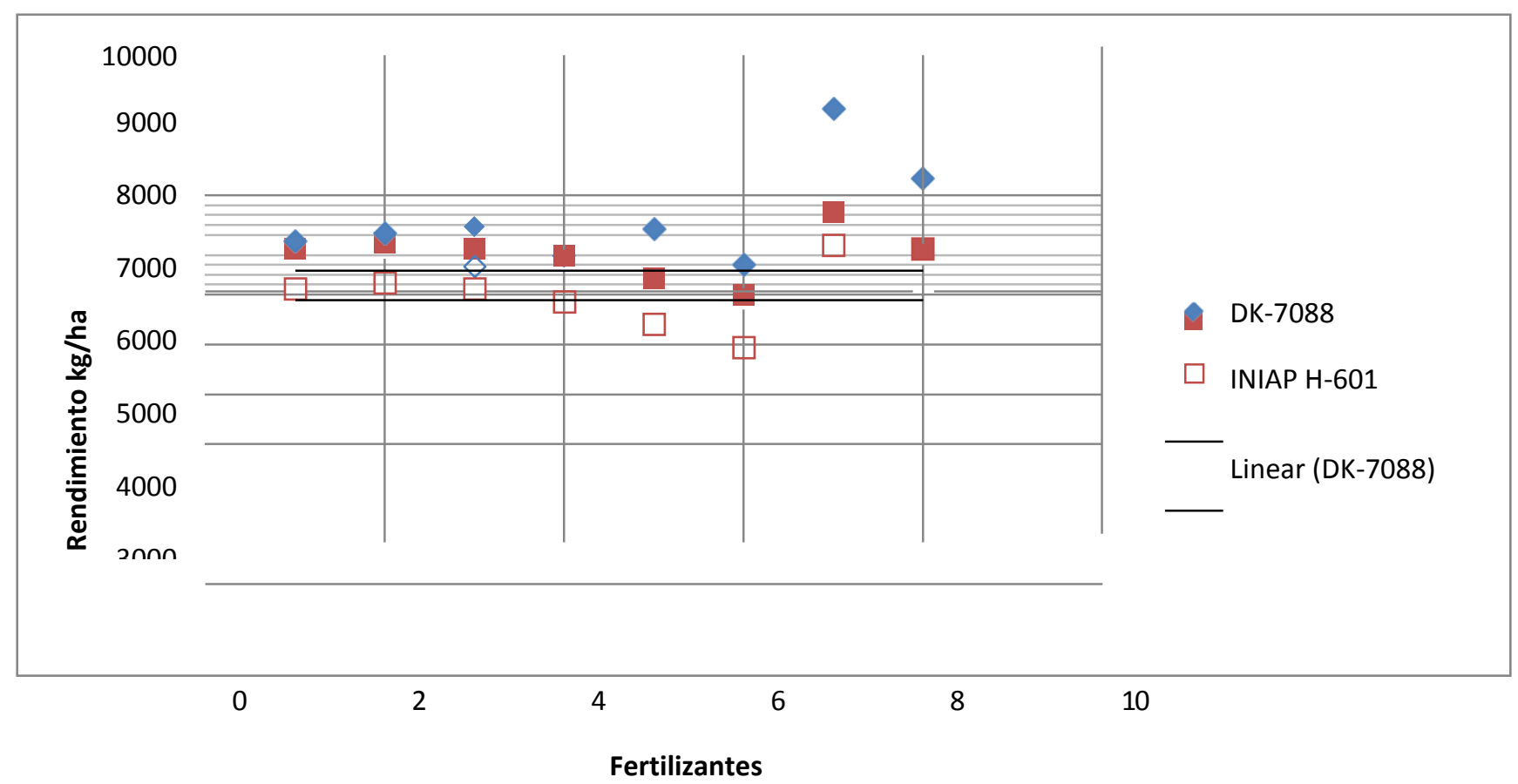

Figura 2. Líneas de tendencia central y dispersión con la aplicación fertilizantes edáficos en mezcla con fertilizantes de liberación controlada. Babahoyo.

\section{DISCUSIÓN Y CONCLUSIONES}

Los resultados muestran que el uso de fertilizantes de liberación controlada solos o en mezclas con fertilización química convencional, influencian el rendimiento del cultivo de maíz en la zona de estudio.

La aplicación de fertilizantes de liberación controlada sobre híbridos de maíz de alto rendimiento, afectan características morfológicas del cultivo, esto es corroborado por Casas (2011) quien manifiesta que las variedades cruzadas o "híbridas" se comportan mejor debido a que la variedad híbrida resultante es más resistente $\mathrm{y}$ productiva.

Los análisis de estadística demuestran que las diferentes aplicaciones de fertilizantes de liberación controlada en conjunto con aplicación de fertilizantes convencionales logran un incremento en las condiciones agronómicas del cultivo de maíz, debido a que los mismos al poner los nutrientes a disposición de la planta de una manera más adecuada y distribuida. Ya que los fertilizantes de liberación proveen la cantidad necesaria de nutrientes para una producción óptima. Además tienen un mínimo efecto negativo sobre el ambiente: el suelo, el agua y la atmósfera. Así en la actualidad hay que llevar a cabo programas de nutrición con criterios variados en la producción y con base analítica de laboratorios, para la corrección de macro y micronutrientes, ya que cada material de siembra tiene sus exigencias peculiares, tanto por la calidad como por la 
cantidad de fertilizantes a aplicar (Melgar, 2012).

Las aplicaciones de este tipo de fertilizante también logran una mejora visual del cultivo, debido a que ellos tienen en su composición química un adecuado balance nutricional, sin embargo este efecto se visualiza mejor comprobando las variables y adicionando fertilizantes que sean de adsorción inmediata. Estos fertilizantes funcionan con programas de manejo avanzado de fertilización y sistemas de cultivo innovadores y contribuyen a la reducción de pérdidas de nitrógeno.

El mayor rendimiento en peso de grano se encontró con la aplicación de fertilización química en un $50 \%$ de la dosis $(70 \mathrm{~kg} / \mathrm{ha} \mathrm{N}+$ $15 \mathrm{~kg} / \mathrm{ha} \mathrm{P}+30 \mathrm{~kg} / \mathrm{ha} \mathrm{K})+$ el $25 \%(112$ $\mathrm{kg} / \mathrm{ha}$ ) de la dosis de Multisuelo. Esta aplicación incentiva al cultivo a lograr un crecimiento relativamente parejo y sostenido, lo cual es previsible ya que la aportación balanceada de nutrientes y su mejor distribución en el sistema radicular estimulan el desarrollo vegetativo adecuado de las plantas maximizando su potencial productivo. Los rendimientos presentados fueron muy aceptables, alcanzando su tope más alto con $7854.2 \mathrm{~kg} / \mathrm{ha}$ en promedio siendo el hibrido de maíz DK-7088 (9041.7 kg/ha) superior a la producción del híbrido H-601 (6666.7 kg/ha). Sin embargo los resultados alcanzados con la utilización de fertilizantes de liberación controlada solos, no superan a la mezcla de estos con fertilización convencional.

\section{REFERENCIAS}

AGRIPAC S.A. 2010. Mixpac, nueva solución para el agro. Revista AGRIPAC DIRECTO, Disponible en www.agripac.com

Casas, F. (2011). Producción Agropecuaria en el Paraguay. In Memoria Seminario-
Taller. 3-6 Nov-2010. IICA-CIID. LimaPerú. 107 p. Disponible en: www.unlm.edu

ENLASA. 2013. Manual y catálogo de productos. Disponible en: www.grupoenlasa.com.ec

FAO (Food and Agriculture Organization of the United Nations). (2006). Guía para identificar las limitaciones de campo en la Producción de maíz. (In Línea). Consultado el 7 enero del 2010 Disponible en www.fao.org/docrep

INIAP, 2008. Evaluación de un vivero de adaptación y rendimiento de 12 híbridos promisorios de maíz. Estación experimental Santa Catalina, Programa de Cereales. Pp. 14 - 15

Melgar, R. (2012). Nuevas Tecnologías en el uso eficiente de fertilizantes nitrogenados. Instituto Nacional de tecnología agropecuaria-INTA. In Simposio Internacional: manejo y uso eficiente de fertilizantes. Buenos Aires. Pp 1-59

Sierra, L.; Simonne, P.; Treadwell, B. 2007. Manejo y rotación de cultivos de cereales, fertilización de los cultivos, Edit MacGraw -Hill, Madrid. Pp 32-39

Sumitomo. 2012. Catalogo y manual de productos. Catálogo 2012, Disponible en www.sumitomo- agricola.com

Thompson, L. M.; Troeh, F.; (2002). Los suelos y su fertilidad. Editorial Reverté, S. A. España. Pp. 229 - 231

Trenkel, W.M. 2007. Fertilizantes y el Ambiente. Instituto Internacional de nutrición de plantas. Informaciones Agronómicas. No 44. pp. 6-7

Tucunango, W. 1993. Nutrición mineral de las plantas. Fitosan S.A. Guayaquil- Ecuador. P 5 